\title{
Sound propagation in a cylindrical Bose-condensed gas
}

\author{
E. Zaremba \\ Department of Physics, Queen's University \\ Kingston, Ontario, Canada K'L 3N6
}

(March 2, 2018)

\begin{abstract}
We study the normal modes of a cylindrical Bose condensate at $T=0$ using the linearized timedependent Gross-Pitaevskii equation in the Thomas-Fermi limit. These modes are relevant to the recent observation of pulse propagation in long, cigar-shaped traps. We find that pulses generated in a cylindrical condensate propagate with little spread at a speed $c=\sqrt{g \bar{n} / m}$, where $\bar{n}$ is the average density of the condensate over its cross-sectional area.
\end{abstract}

PACS numbers: 03.75.Fi, 67.40.Hf, 67.57.Jj

In a recent paper, Andrews et al. [1] presented results for the propagation of sound through the Bose condensate in an axially-symmetric, cigar-shaped trap. These observations were made possible by the large aspect ratio of the anisotropic trap and the localized generation of pulses by a laser beam focused at the mid-point along the length of the trap. By imaging the propagation of the pulse, they were able to determine the sound speed as a function of the condensate density. The measured sound speed is approximately equal to the Bogoliubov phonon velocity as determined by the maximum density in the cloud. The authors [1] sketch a qualitative theoretical argument in support of this observation.

The purpose of the present paper is to provide a more detailed analysis of the dependence of the sound speed on the inhomogeneous distribution of atoms in the trap. In the propagation of a pulse for short times, the ends of the trapped condensate do not come into play and we can therefore simulate the experimental situation by considering an idealized cylindrical trap which is uniform in the $z$-direction and which has a harmonic confining potential of the form

$$
V(\rho)=\frac{1}{2} m \omega_{0}^{2} \rho^{2}
$$

in the radial direction. Here, $\omega_{0}$ is the trap frequency. Treating the condensate in the Thomas-Fermi (TF) approximation [2], the equilibrium density is given by

$$
n_{0}(\rho)=\frac{m \omega_{0}^{2}}{2 g}\left(R^{2}-\rho^{2}\right),
$$

where $g=4 \pi a \hbar^{2} / m$ is the interaction parameter and $R$ is the radius of the cylindrical condensate related to the chemical potential, $\mu=V(\rho)+g n_{0}(\rho)$, by $\mu=\frac{1}{2} m \omega_{0}^{2} R^{2}$.

The dynamics of the condensate will be based on the linearized time-dependent Gross-Pitaevskii equation [3] in the TF limit 迎. This equation can be recast as a pair of quantum hydrodynamic equations

$$
\begin{aligned}
\frac{\partial \delta n}{\partial t} & =-\nabla \cdot\left(n_{0} \mathbf{v}\right) \\
m \frac{\partial \mathbf{v}}{\partial t} & =-g \nabla \delta n-\nabla \delta U,
\end{aligned}
$$

where $\delta U(\mathbf{r}, t)$ represents an externally imposed potential. Eliminating the velocity from these equations, we obtain an equation for the density fluctuation $\delta n(\mathbf{r}, t)$ first derived (for $\delta U=0$ ) by Stringari [4],

$$
\frac{\partial^{2} \delta n}{\partial t^{2}}=\frac{g}{m} \nabla \cdot\left(n_{0} \nabla \delta n\right)+\frac{1}{m} \nabla \cdot\left(n_{0} \nabla \delta U\right) .
$$

To study the normal modes of the condensate, we set $\delta U$ to zero and look for solutions which correspond to a propagating wave of the form

$$
\delta n(\mathbf{r}, t)=\delta n(\rho) e^{i(k z-\omega t)} .
$$

We assume that the density fluctuation in the transverse directions depends only on the radial variable $\rho$ and not on the azimuthal angle $\phi$. It is clear that more general solutions having the angular dependence $e^{i m \phi}$ are possible, but these will not be considered in this paper.

We now substitute this form of the solution into (5) and, making use of the equilibrium density in (2), obtain the equation $(\delta U=0)$

$$
\begin{gathered}
\omega^{2} \delta n=\frac{1}{2} \omega_{0}^{2}\left\{k^{2}\left(R^{2}-\rho^{2}\right) \delta n-\frac{1}{\rho}\left(R^{2}-3 \rho^{2}\right) \frac{\partial \delta n}{\partial \rho}\right. \\
\left.-\left(R^{2}-\rho^{2}\right) \frac{\partial^{2} \delta n}{\partial \rho^{2}}\right\}
\end{gathered}
$$

where $\delta n$ now represents the spatially-dependent amplitude defined in (6). The allowed solutions of this equation define the dispersion relations, $\omega(k)$, of the various modes of the condensate in the cylindrical trap.

It is now convenient to introduce the dimensionless parameters

$$
\bar{\omega} \equiv \frac{\omega}{\omega_{0}}, \quad \bar{k} \equiv k R
$$

and to define the new independent variable

$$
x \equiv 2 \frac{\rho^{2}}{R^{2}}-1, \quad-1 \leq x \leq 1 .
$$

When expressed in terms of this variable, the density fluctuation, now denoted by $y(x)$, is found to satisfy 


$$
\frac{d}{d x}\left[\left(1-x^{2}\right) \frac{d y}{d x}\right]+\frac{1}{2} \bar{\omega}^{2} y-\frac{1}{8} \bar{k}^{2}(1-x) y=0,
$$

which is in the standard Sturm-Liouville form. For $\bar{k}=0$, this equation in fact reduces to the Legendre differential equation

$$
\frac{d}{d x}\left[\left(1-x^{2}\right) \frac{d y}{d x}\right]+\lambda y=0
$$

whose solutions are the Legendre polynomials $P_{l}(x)$ with eigenvalues $\lambda=l(l+1), l=0,1, \ldots$ Thus the modes of the condensate can be labeled by the index $l$ and have a limiting frequency at $k=0$ given by

$$
\omega_{l}^{2}(k=0)=2 l(l+1) \omega_{0}^{2} .
$$

The first two frequencies in the sequence, 0 and $2 \omega_{0}$, are the two $m=0$ mode frequencies found by Stringari [4] for a cigar-shaped trap in the limit that the axial frequency, $\omega_{z}$, tends to zero. More generally, the whole sequence in (11) was recently obtained analytically in this same limit by Fliesser et al. [5].

These various solutions have distinct radial eigenfunctions. For example, for $l=0, y(x)=$ a constant, and the associated density fluctuation has a radially independent amplitude. This mode corresponds to a local adiabatic expansion of the condensate brought about by a local change in the chemical potential, $\delta \mu$. Such a change gives rise to a variation in the equilibrium density $n_{0}(\rho ; R)$ which is spatially independent. In the limit of long wavelengths, it costs no energy to move atoms from one region of the trap to another and the mode therefore has zero frequency. The other $l \neq 0$ modes have a finite frequency since they involve radial motion of the condensate. (Note that according to (4), the direction of the local velocity is normal to constant density surfaces.) For example, for $l=1$, the density fluctuation is of the form $\delta n(\rho) \propto\left(1-2 \rho^{2} / R^{2}\right)$ which has a node at $\rho=R / \sqrt{2}$. Unlike the $l=0$ mode, all of these modes have a number conserving density fluctuation in the sense that $\int_{0}^{R} d \rho \rho \delta n(\rho)=0$. The $l=1$ mode at $k=0$ is a radial breathing mode.

For $k \neq 0$, we expand the density fluctuation as

$$
y(x)=\sum_{l} a_{l} P_{l}(x)
$$

where the normalization of the Legendre functions is chosen such that

$$
\int_{-1}^{1} d x P_{l}(x) P_{l^{\prime}}(x)=\delta_{l l^{\prime}}
$$

Substituting expansion (12) into (9), we obtain the system of linear equations

$$
\left(\frac{1}{2} \bar{\omega}^{2}-l(l+1)-\frac{1}{8} \bar{k}^{2}\right) a_{l}+\frac{1}{8} \bar{k}^{2} \sum_{l^{\prime}} M_{l l^{\prime}} a_{l^{\prime}}=0,
$$

where the symmetric matrix $M_{l l^{\prime}}$ is given by

$$
M_{l l^{\prime}}=\int_{-1}^{1} d x P_{l}(x) x P_{l^{\prime}}(x)
$$

Since the matrix $M_{l l^{\prime}}$ does not have diagonal matrix elements, it is clear that its effect on the mode eigenvalues first occurs in second order, so that to lowest order in $k^{2}$,

$$
\omega_{l}^{2}(k)=2 l(l+1) \omega_{0}^{2}+\frac{1}{4}\left(k R \omega_{0}\right)^{2}+O\left(k^{4}\right) .
$$

In particular, the $l=0$ mode is phonon-like with a velocity

$$
c=\frac{1}{2} R \omega_{0}=\sqrt{\frac{g n_{0}(0)}{2 m}},
$$

where $n_{0}(0)$ is the (maximum) condensate density on the axis of the trap. If we compare this velocity with the Bogoliubov velocity $\sqrt{g n / m}$ in a homogeneous gas of density $n$, we see that the effective density in the cylindrical trap is half the maximum value, which is just the density, $\bar{n}$, obtained by averaging (2) over the condensate cross-section.

It is interesting to note that the matrix problem in (14) is equivalent to the problem of a quantum rigid rotor in a gravitational field, having the Hamiltonian

$$
H=\frac{L^{2}}{2 I}+M g R(1-\cos \theta) .
$$

Here, $L$ is the angular momentum operator, $I=M R^{2}$ is the moment of inertia of the rotor and the gravitational potential energy of the rotor mass $M$ is referenced with respect to its lowest position (the polar angle $\theta$ is defined with respect to this position). The angular eigenfunctions of this Hamiltonian can be expanded in spherical harmonics $Y_{l m}(\theta, \phi)$ and in the $m=0$ subspace, the problem is then identical to (14). Thus the parameter $\bar{k}$ plays the role of a 'gravitational field' which perturbs the mode energies from their 'free rotor' values.

The solution of (14) is easily obtained for arbitrary $\bar{k}$ by numerical diagonalization. In Fig. 1 we show some of the lowest mode frequencies as a function of wavevector. The lowest mode is a sound-like mode and exhibits a negative dispersion. It can be seen that the group velocity deviates appreciably from its long wavelength limit once the wavelength is comparable to the diameter of the condensate. The mode-coupling induced by the $\bar{k}^{2}$ perturbation in (14) of course becomes more significant with increasing $\bar{k}$ and has the effect of lowering the sound speed. This coupling is associated with the interplay of the wave-like modulation of the density along the axis and the strong inhomogeneity of the equilibrium density in the radial direction.

We now return to the experimental situation [1] which involves the propagation of sound pulses rather than continuous waves. These pulses are generated by switching 
on a laser beam which repels atoms from its point of application at the center of the cylindrical trap. We shall assume this perturbation to be weak and consider the linear response of the condensate. The perturbation is taken to be

$$
\delta U(\mathbf{r}, t)=U_{0} e^{-z^{2} / \sigma^{2}} \theta(t),
$$

that is, a gaussian potential with no radial dependence, which is switched on at $t=0$. The equation defining the density fluctuation is given by (5) and can be solved by introducing a Fourier representation of the density and external potential:

$$
\begin{aligned}
\delta n(\rho, z, t) & =\int_{-\infty}^{\infty} \frac{d k}{2 \pi} e^{i k z} \delta n(\rho, k, t) \\
\delta U(z, t) & =\int_{-\infty}^{\infty} \frac{d k}{2 \pi} e^{i k z} \delta U(k, t)
\end{aligned}
$$

with

$$
\delta U(k, t)=\sqrt{\pi} \sigma U_{0} e^{-\sigma^{2} k^{2} / 4} \theta(t) \equiv \delta U(k) \theta(t) .
$$

Taking the Fourier transform of (5) and using the variable $x$ introduced earlier, we obtain the equation

$$
\frac{\partial^{2} y}{\partial t^{2}}=-\hat{L} y-\frac{\left(\omega_{0} k R\right)^{2}}{4 g}(1-x) \delta U
$$

where we have defined the differential operator

$$
\hat{L} \equiv-2 \omega_{0}^{2}\left[\frac{d}{d x}\left(1-x^{2}\right) \frac{d}{d x}-\frac{1}{8} \bar{k}^{2}(1-x)\right] .
$$

This is just the differential operator defining the mode eigenfunctions in (9). These eigenfunctions satisfy

$$
\hat{L} \phi_{m}(x)=\omega_{m}^{2}(k) \phi_{m}(x)
$$

and form an orthonormal set,

$$
\int_{-1}^{1} d x \phi_{m}(x) \phi_{n}(x)=\delta_{m n} .
$$

They can therefore be used to expand $y(x, k, t)$ as

$$
y(x, k, t)=\sum_{m} b_{m}(k, t) \phi_{m}(x) .
$$

Substituting this expansion into (22) and taking the inner product of the resulting equation with $\phi_{n}(x)$, we obtain the equation for a driven harmonic oscillator

$$
\ddot{b}_{n}+\omega_{n}^{2} b_{n}=f_{n}(t)
$$

where the forcing term on the right hand side is defined as

$$
\begin{aligned}
f_{n}(t) & =-\frac{\left(\omega_{0} k R\right)^{2}}{4 g} \delta U(k, t) \int_{-1}^{1} d x \phi_{n}(x)(1-x) \\
& \equiv f_{n}(k) \theta(t)
\end{aligned}
$$

The solution of (27) for $t \geq 0$ with the boundary conditions $b_{n}(0)=0$ and $\dot{b}_{n}(0)=0$ is

$$
b_{n}(k, t)=\frac{f_{n}(k)}{\omega_{n}^{2}(k)}\left[1-\cos \omega_{n}(k) t\right] .
$$

We have here explicitly displayed the dependence of the various quantities on the wavevector $k$ in the Fourier expansion. This essentially completes the solution for the density fluctuation $\delta n(\rho, z, t)$.

To analyze the time evolution of the density pulse, it is convenient to consider the average of $\delta n(\rho, z, t)$ over the cross-sectional area, $A$, of the condensate. We therefore define

$$
\begin{aligned}
\overline{\delta n}(z, t) & =\frac{1}{A} \int_{A} d A \delta n(\rho, z, t) \\
& =\int_{-\infty}^{\infty} \frac{d k}{2 \pi} e^{i k z} \sum_{n} b_{n}(k, t) \frac{1}{2} \int_{-1}^{1} d x \phi_{n}(x) .
\end{aligned}
$$

According to (12), the eigenfunctions $\phi_{n}(x)$ are themselves given by the expansion

$$
\phi_{n}(x)=\sum_{l} a_{l}^{(n)}(k) P_{l}(x),
$$

where the coefficients $a_{l}^{(n)}(k)$ defining the $n$-th eigenvector of (14) have the limiting value $a_{l}^{(n)}(k=0)=\delta_{l n}$. (In other words, the eigenfunction $\phi_{n}(x)$ reduces to $P_{n}(x)$ in the $k \rightarrow 0$ limit.) The orthonormality of the Legendre functions thus yields

$$
\overline{\delta n}(z, t)=\int_{-\infty}^{\infty} \frac{d k}{2 \pi} e^{i k z} \frac{1}{\sqrt{2}} \sum_{n} a_{0}^{(n)}(k) b_{n}(k, t) .
$$

Similarly, we find

$$
f_{n}(k)=-\frac{\left(\omega_{0} k R\right)^{2}}{2 \sqrt{2} g}\left(a_{0}^{(n)}(k)-\frac{1}{\sqrt{3}} a_{1}^{(n)}(k)\right) \delta U(k) .
$$

Thus, for small $k$, only the $n=0$ and $n=1$ modes are excited appreciably.

Substituting (29) together with (33) into (32), the averaged density fluctuation can be evaluated numerically. As an example of the calculation, we present in Fig. 2 results for the propagation of a pulse. The width of the gaussian perturbation in these calculations was chosen to be $\sigma=1.5 R$, which corresponds approximately to the experimental situation. Once the pulse is launched it is seen to propagate at the speed $c$ given by $(17)$. The shape of the pulse will change in time as a result of the $k$-dispersion of the normal modes, but the effect is weak since the pulse is mainly made up of the long-wavelength $n=0$ sound modes. The particles carried off by the pulse leave behind a static depression at the origin which corresponds to the new equilibrium shape of the condensate in the presence of the applied localized perturbation. 
These theoretical results for the propagation of a pulse are in reasonable agreement with the experimental observations [1]. One difference is the stronger wave packet dispersion observed experimentally which may be due to the actual variation of the condensate density along the trap axis. However, a more serious discrepancy concerns the speed of propagation. In the experiment it was concluded that the sound speed was determined by the maximum condensate density according to $c^{\prime} \simeq \sqrt{g n_{0}(0) / m}$, as opposed to the value we find in (17). However it is clear from the published figure that the measured sound speed actually lies below $c^{\prime}$ at the higher trap densities. At these densities, the theoretical value $c$ would lie below the measured values by about the same amount that $c^{\prime}$ lies above. The difference between our theoretical result and experiment could be explained by errors in the determination of the trap density on which the theoretical value is based, but the claimed experimental precision (W. Ketterle, private communication) would seem to eliminate this possibility.

On the theoretical side, one can question the validity of the TF approximation. For the given trap parameters [1] and a typical density of $n_{0}(0) \sim 3 \times 10^{20} \mathrm{~m}^{-3}$, the radius of the condensate $R$, in units of the oscillator length $l=\sqrt{\hbar / m \omega_{0}}$, is approximately 5 . This implies that the TF approximation is providing a good estimate of the ground state density over most of the occupied volume. The validity of the TF approximation is further supported by the fact that the frequencies of the lowlying collective excitations in these traps [6] are close to the TF values [4]. This observation is consistent with calculations based on the Bogoliubov approximation [7] which show a reasonably rapid convergence to the TF limit with increasing $N$, the number of trapped atoms. However, the situation with regard to pulse propagation is somewhat different in that shorter wavelength excitations are being probed. Within the hydrodynamic formulation [4] of the Bogoliubov approximation, an additional term appears on the right hand side of (4) which represents the fluctuation in the 'quantum pressure' associated with the kinetic energy. This term becomes increasingly important as the wavelength of the density fluctuation is reduced. Unfortunately, it cannot be estimated using the $\mathrm{TF}$ density since the failure of the TF approximation at the edge of the condensate [2, 10] leads to a divergent result. Thus, explicit numerical solutions of the Bogoliubov equations are required in order to ascertain the accuracy of the TF approximation for the conditions under which the pulse propagation experiments were performed. On the other hand, at long wavelengths, we expect the TF prediction for the sound speed, as given by (17), to be valid.

This work was supported by a grant from the Natural Sciences and Engineering Research Council of Canada. I would like to thank Dr. A. Griffin for a critical reading of the manuscript and Dr. W. Ketterle for useful discussions. I would also like to acknowledge the hospitality of Il Ciocco where this work was begun.
[1] M.R. Andrews, D.M. Kurn, H.-J. Miesner, D.S. Durfee, C.G. Townsend, S. Inouye and W. Ketterle, Phys. Rev. Lett. 79, 553 (1997).

[2] G. Baym and C.J. Pethick, Phys. Rev. Lett. 76, 6 (1996).

[3] L.P. Pitaevskii, Zh. Eksp. Teor. Fiz. 40, 646 (1961) [Sov. Phys. JETP 13, 451 (1961)]; E.P. Gross, Nuovo Cimento 20, 454 (1961); J. Math. Phys. 4, 195 (1963).

[4] S. Stringari, Phys. Rev. Lett. 77, 2360 (1996).

[5] M. Fliesser, A. Csordás, P. Szépfalusy and R. Graham, preprint, cond-mat/9706002

[6] M.-O. Mewes, M.R. Andrews, N.J. van Druten, D.M. Kurn, D.S. Durfee, C.G. Townsend and W. Ketterle, Phys. Rev. Lett. 77, 988 (1996).

[7] K. G. Singh and D. S. Rokhsar, Phys. Rev. Lett. 77, 1667 (1996).

[8] M. Edwards, P.A. Ruprecht, K. Burnett, R.J. Dodd and C.W. Clark, Phys. Rev. Lett. 77, 1671 (1996).

[9] V.M. Pérez-García, H. Michinel, J.I. Cirac, M. Lewenstein and P. Zoller, Phys. Rev. Lett. 77, 5320 (1996).

[10] F. Dalfovo, L.P. Pitaevskii and S. Stringari, Phys. Rev. A 54, 4213 (1996).

\section{FIGURE CAPTIONS}

Fig.1: The dispersion of the lowest modes in a cylindrical condensate as a function of the wavevector $k$. $\omega_{0}$ is the radial trap frequency and $R$ is the TF condensate radius.

Fig.2: The propagation of a pulse generated by a gaussian perturbation applied at the origin at $t=0$. The lowest curve is at a time $t=4 / \omega_{0}$, and the interval between each successive curve is $\Delta t=4 / \omega_{0}$. 


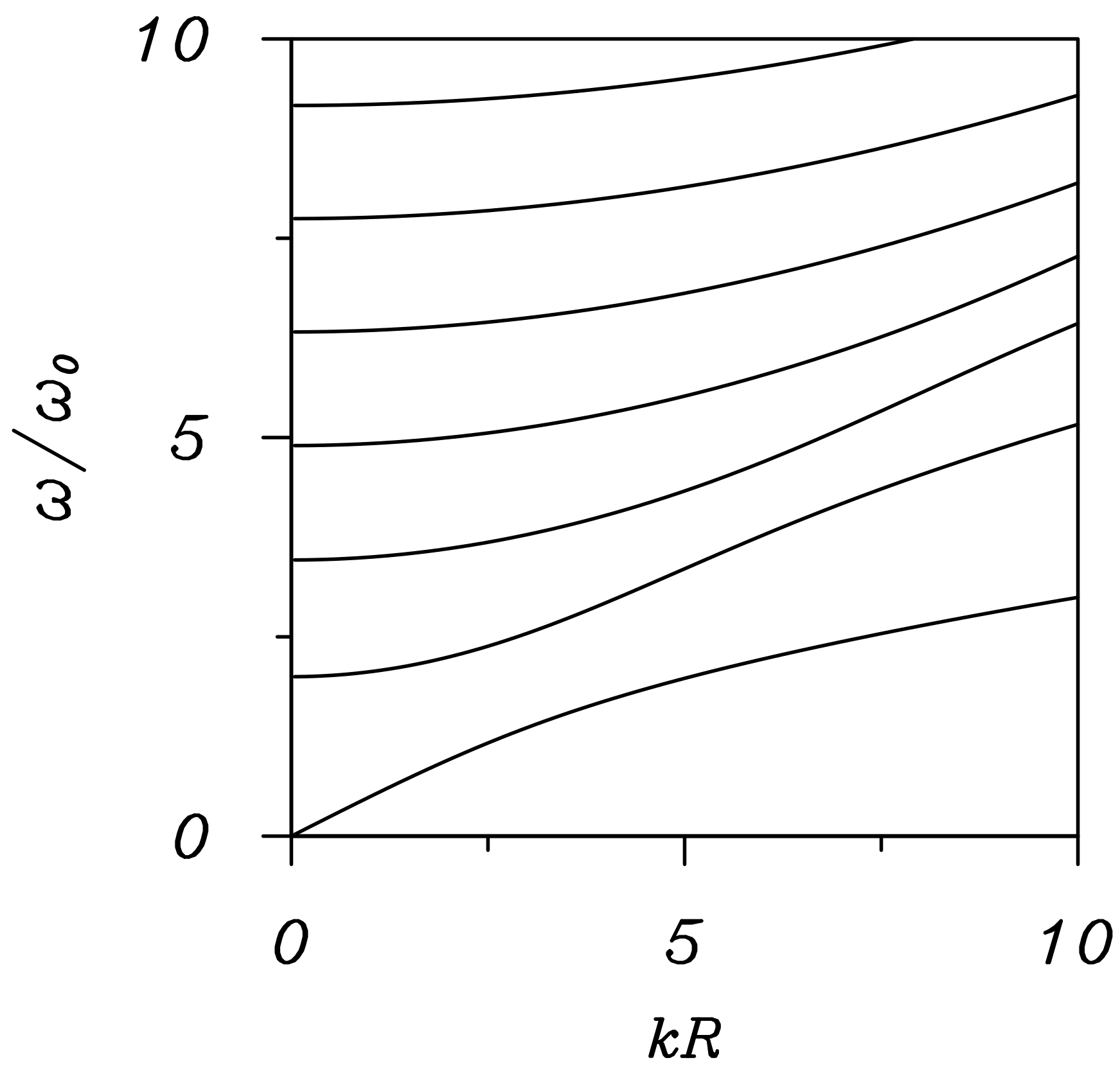




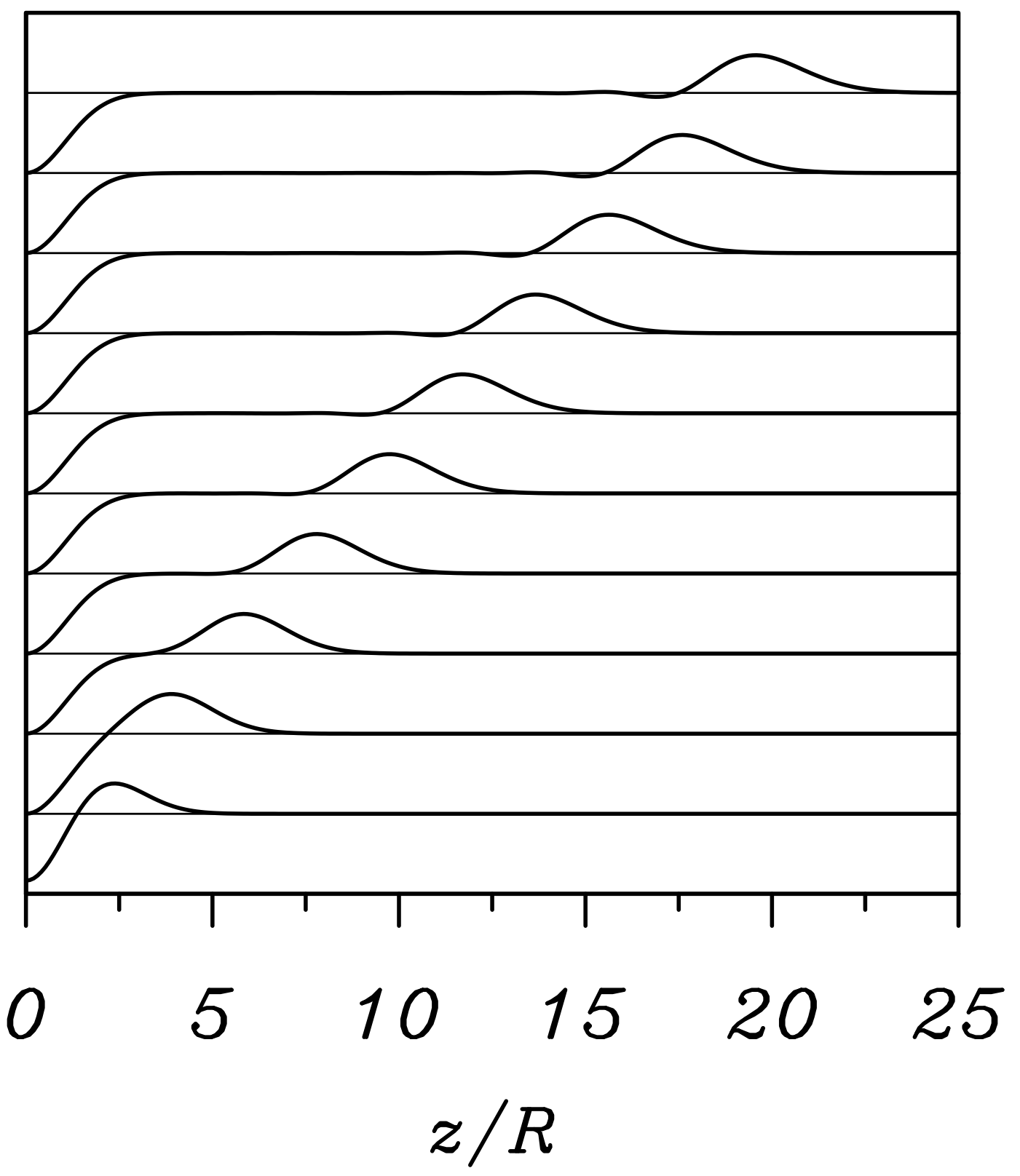

